\title{
THE CURRENT KNOWLEDGE OF INVERTEBRATE AQUAPORIN WATER CHANNELS WITH PARTICULAR EMPHASIS ON INSECT AQPS
}

\author{
Ewa TOMKOWIAK, Joanna Romana PIENKOWSKA
}

\author{
Department of Cell Biology, Faculty of Biology, Adam Mickiewicz University
}

\author{
DOI: $10.2478 / \mathrm{v} 10052-010-0005-7$
}

\begin{abstract}
Summary: Aquaporins (AQPs) or water channels are some of the most ubiquitous integral membrane proteins, and are present in all living organisms. Their presence in the lipid bilayer of cell membranes considerably increases their permeability to water and, in some cases, to other small solutes. All AQPs, identified thus far, share the same structure, comprising of six transmembrane segments and two conserved regions forming the pore. Depending on the transported solutes, AQPs can be divided into two classes: 'classical' aquaporins (permeable only to water) and aquaglyceroporins (permeable also to glycerol and/or other solutes). Many subtypes of AQPs coexist in a single organism. Localization of particular subtypes of AQPs is tissue-specific. AQPs have been well characterized in almost all vertebrate classes. However, little is known about their counterparts in invertebrates. Most of the water channels characterized in invertebrates are found in insects. Therefore, the knowledge of aquaporins in invertebrates is generally limited to the information concerning water channels in this class of organism. Insects are characterized by an astonishing variety of physiological adaptations, notable in their feeding strategies or survival strategies in hostile environments. An example of such, is feeding on blood, or tolerating extreme cold or drought. It is likely that many of these adaptation patterns emerged due to the expression and regulation of particular aquaporins. Here we review the current state of knowledge of invertebrate AQPs (of insects and nematodes) and compare their structure and function with mammalian water channels.
\end{abstract}

Keywords: water channel, aquaporin, cell membrane, water, invertebrates

\section{INTRODUCTION}

In 1992, Peter Agre [1] first described a protein which forms a water channel in a blood cell membrane. It has become clear that this protein, called aquaporin (AQP), is ubiquitous throughout plant [14] and animal [29, 10] kingdoms, as well as among microorganisms [11], filamentous fungi [30], and 
protozoa [4]. This wide-spread occurrence is evidence for the important role of aquaporins. Water channels are divided into two subfamilies based on their permeability properties: primarily water selective aquaporins and aquaglyceroporins (transport small molecules such as glycerol and urea, in addition to water) [23]. Both exhibit a common structure [31] and are members of the major intrinsic protein (MIP) superfamily. Functional AQPs form homotetramers in a plasma membrane. Each monomer consists of one polypeptide chain of approximately $30 \mathrm{kDa}$ with six, membrane spanning, $\alpha$-helical domains. Domains are connected by five loops: three extracellular (A, C, E) and two intracellular (B and D). The loops B and E contain NPA motives which interact together forming a water pore (Fig. 1). The amino acid residues around the loops are responsible for the selectivity of the pore [23]. The $\mathrm{N}$ - and C-terminal ends of the polypeptide are located on the cytoplasmic side of the plasma membrane. The four monomers form a water channel with the four water pores [6] (Fig. 2).

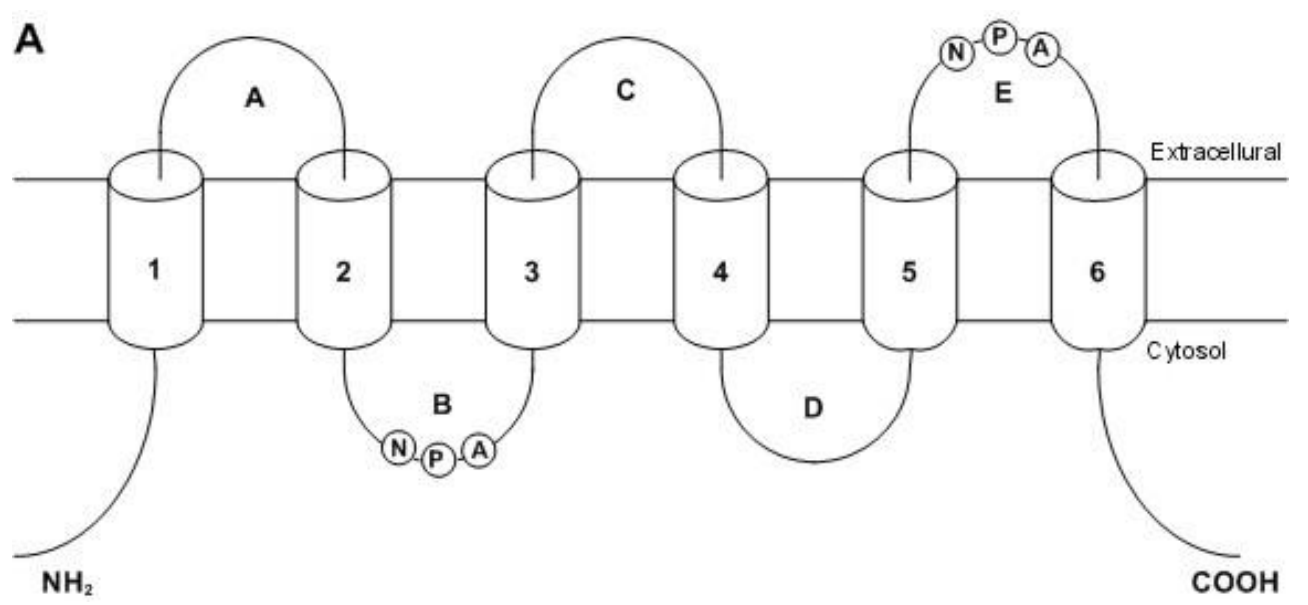

FIGURE 1A. A topology model of AQP1. Transmembrane regions are indicated by numbers and loops by letters. There are highlighted conservative motives NPA in loops B and E (based on [23] )

There are 13 different AQPs (AQP0-AQP12) identified in human cells [22]. The expression patterns of aquaporin genes are tissue-specific and thus are the likely explanation for such a great number of different aquaporin-built water channels identified in each organism [34]. AQPs0-8 are primarily water selective aquaporins, whereas AQP3, AQP7, AQP9, and AQP10 are aquaglyceroporins. There is insufficient knowledge about AQP11 and AQP12 to classify these proteins into the above two groups [22]. Researchers have recently reported that aquaporins are capable of conducting unconventional permeates such as $\mathrm{CO}_{2}, \mathrm{H}_{2} \mathrm{O}_{2}, \mathrm{NO}, \mathrm{NO}_{2}$ and $\mathrm{NH}_{3}$ [32]. The significance of this transport for the functioning of cells and tissues is not yet clear [32]. 


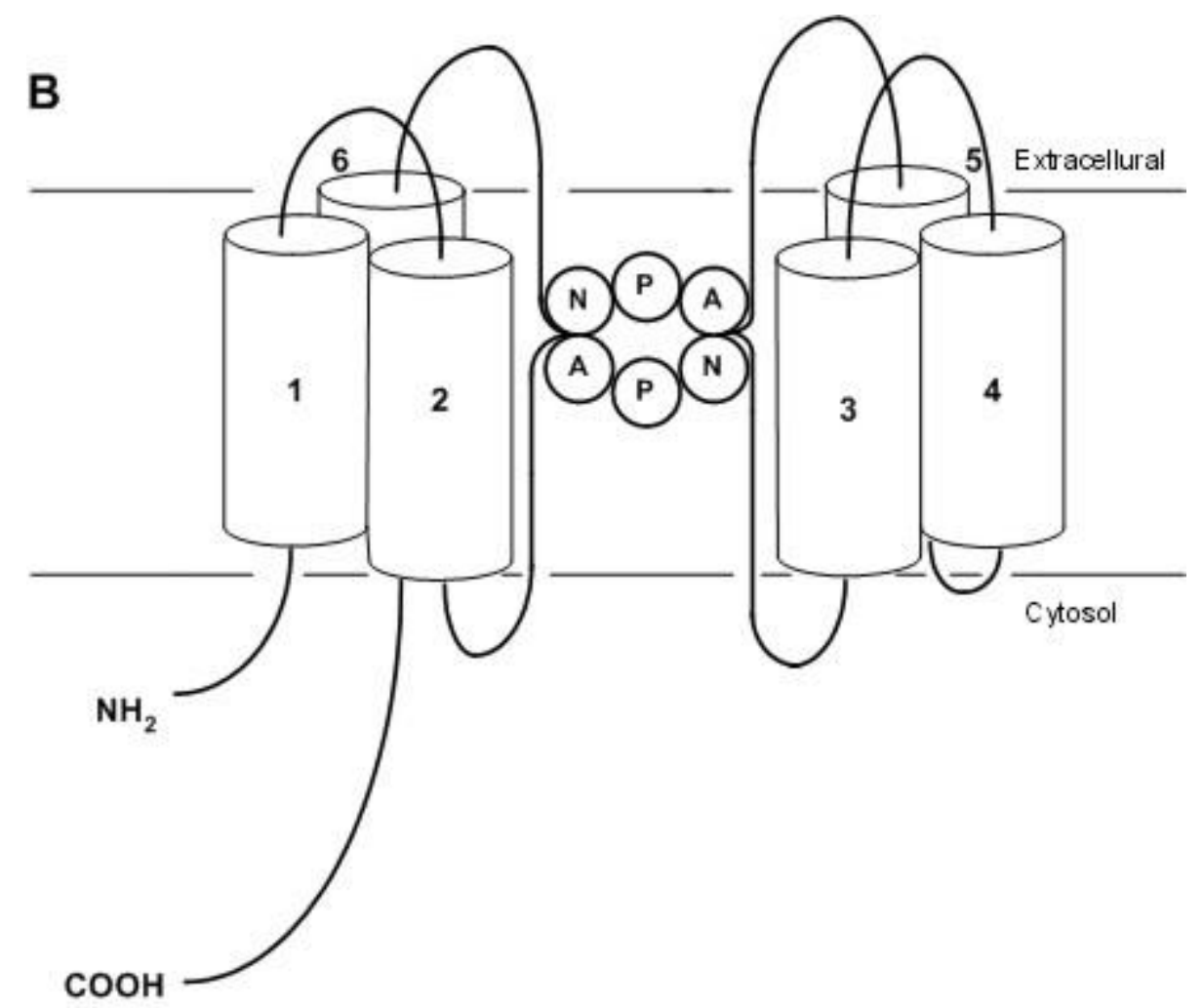

FIGURE 1B. An hourglass structure of aquaporin in the bilayer (based on [6] )

Aquaporins have been identified in organs and tissues of almost all vertebrates, where they share a common structure but could have different functions [21, 25, 28], however invertebrate aquaporins have not yet been as well characterized. There have been to date only a few water channels described in insects and nematodes.

\section{INSECT AQUAPORINS}

In all cases where insects have been tested for the presence of aquaporins, researchers were only able to identify one type of a water channel for each species. However, multiple aquaporins have been found in Drosophila melanogaster.

\section{Aquaporin identified in mosquito Aedes aegypti}

Only one aquaporin gene was found in the mosquito Aedes aegypti, which is expressed in the respiratory system. This location is connected with the 


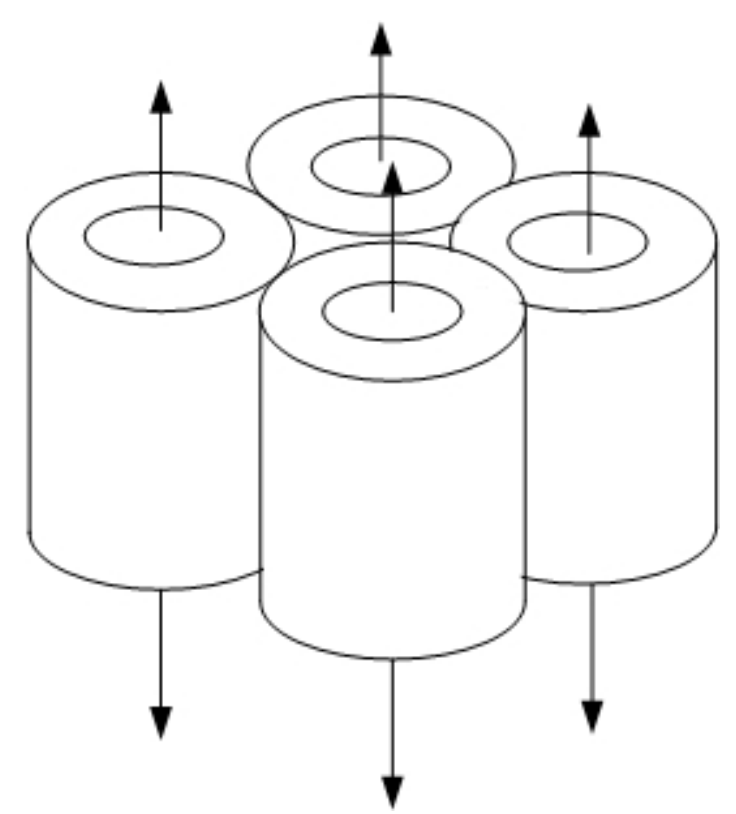

FIGURE 2. Tetrameric assembly of AQP1 in a membrane in which individual monomers contain water pores (based on [6] )

functioning of the tracheole system. Very important to this system, is regulation of fluid volume in the ends of the tracheoles. The fluid volume depends on the metabolic activity of the tissues. This mechanism regulates the diffusion rate from the tracheoles to the oxygen-demanding cells. During periods of high activity, the fluid volume decreases and oxygen can reach cells faster. Alternatively, at rest, the fluid volume increases and the rate of diffusion is slower. The aquaporin located in the tracheole ends has been called AeaAQP [24]. The water channel built by this aquaporin takes part in the regulation of the fluid volume in the respiratory system [24]. An important feature is that aquaporins have been found in the ends of tracheoles are closely associated with the Malpighian tubules. Furthermore, a transcript of $A e a A Q P$ is localized in the insect's hingut cells which are responsible for oxygen exchange. However, proteins built a water channel were not detected. In conclusion, it seems that the AeaAQP is localized in a part of the respiratory system, and is responsible for the regulation of the fluid volume in the ends of the tracheoles, which supplies the Malpighian tubules with oxygen [5, 7].

Expression of aquaporin (AeaAQP) in Xenopus laevis oocytes is the best method for investigating water channel properties [7]. It has been demonstrated that the rate of water transport through the plasma membrane increases 10-fold in the presence of the aquaporin. The permeability of the water channel was inhibited by mercury ions. Transport of glycerol, urea, and other small solutes was not seen. Furthermore, this experiment showed that this protein forms crystalline orthogonal 
arrays of particles (OAPs) in the plasma membrane. Particle spacing within OAPs was $6.8-6.9 \mathrm{~nm}$. This value is comparable to spacing within OAPs formed by aquaporins in other organisms. Aquaporins were not observed outside this structure [7].

AeaAQP is a protein composed of 249 residues $(26.1 \mathrm{kDa})$ [24]. Comparison of this amino acid sequence with well-known proteins of water channels in other organisms allows the following features to be concluded: (1) the sensitivity of the mercury ions, as previously mentioned, which can inhibit transport through the water pore, (2) responsibility of the binding of mercury ions, and as a consequence, the blockage of water permeability, is likely caused by a cysteine at position 79 localized near the NPA motif in loop B (Fig. 3). Based on the amino acid sequence, it can be concluded that this protein is kinase independent (lacks sequence Arg-Arg-X-Ser), as it lacks a phosphorylation site. On the other hand, there is a potential N-glycosylation site in loop E (Asn211-Trp212-Thr213) [24].

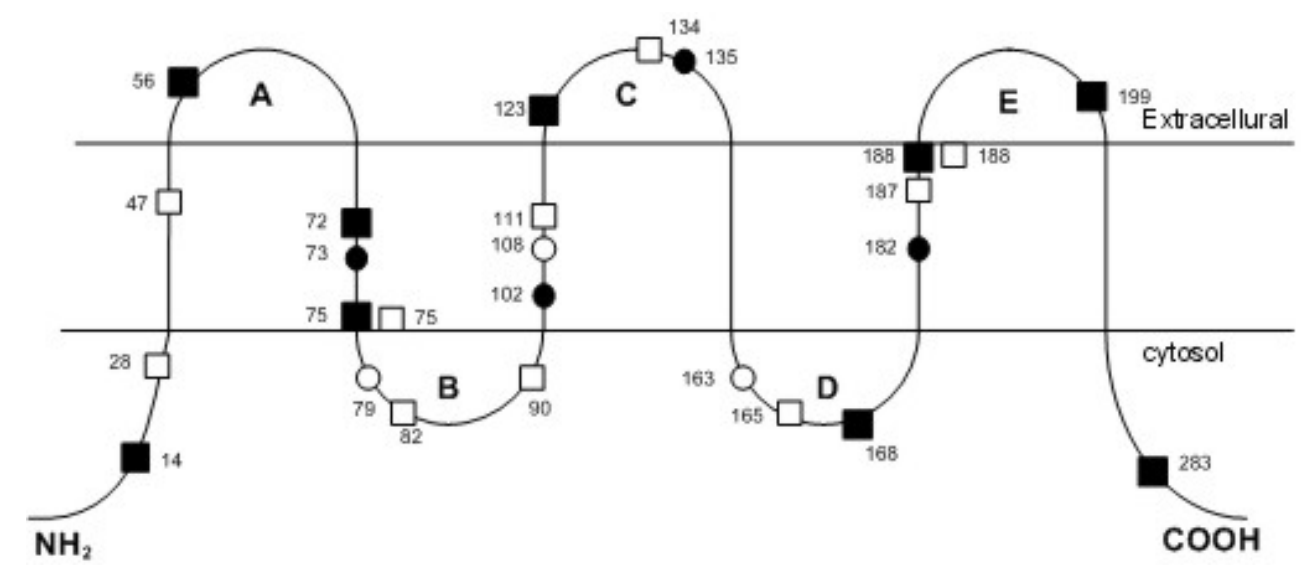

FIGURE 3. Localization of cysteines in polypeptide chain of aquaporin: A.aegypti ${ }^{\bigcirc}$, R.prolixus C.viridis $\square$ i DRIP D.melanogaster (based on [24] )

Aquaporin identified in heteroptera Rhodnius prolixus

Rhodnius prolixus belongs the heteroptera species, that periodically ingest meals overcoming many times their own size. A portion of the ingested volume must be eliminated in a short time. This process starts 10-15 minutes after feeding with the secretion of a diuretic hormone (DH) by ganglia cells that are stimulated by an increased volume of the insect's abdomen. DH induces fluid secretion by acting on the Malpighian tubules. This observation suggests that proteins founded in the cells of the Malpighian tubules can be classified to the aquaporin family. Research has confirmed the presence of aquaporins in these cells. The isolated 
protein was named Rp-MIP after the insect and major intrinsic protein superfamily [8].

In order to investigate the water movement through Rp-MIP, mRNA for this protein was expressed in X. laevis oocytes [8]. When compared with control oocytes, transport through the oocyte cell membrane, with an injected aquaporin mRNA, increases 2 -fold. It should be noted that in comparison with other known aquaporins this is a small increase. The sensitivity of Rp-MIP to mercury ions has also been evaluated. Treating the oocytes with mercury ions did not reduce transport of water, suggesting that Rp-MIP is not sensitive to mercury ions, despite having nine cysteines in the amino acid sequence that could potentially bind these ions (Fig. 3).

Based on $R p-M I P$ gene structure, it is possible to suppose that the product of this gene is a protein which consists of 286 residues $(31 \mathrm{kDa})$. As in all known aquaporins, the $\mathrm{N}$ - and $\mathrm{C}$-terminus are localized in the cytoplasm and the polypeptide chain forms six transmembrane domains. Unlike other aquaporins, RpMIP has the NPV motif (Asn-Pro-Val) in loop E instead of the typical NPA motif (Asn-Pro-Ala). The amino acid sequence indicates that Ser11 is a phosphorylation site of the cyclic adenosine monophosphate - dependent protein kinase (PKA). Ser25 and Ser262 are potential phosphorylation sites for another kinase, kinase CaM. Asn248 is a potential site for N-glycosylation [8].

\section{Aquaporin localized in homopteran sap-sucking insect Cicadella viridis}

Research of aquaporins in Cicadella viridis began with the filter chamber [18], a structure, built by highly specialized epithelial cells, localized in the digestive tract. It is responsible for the rapid transport of water from the initial midgut, to the terminal midgut and Malpighian tubules. mRNA isolated from the epithelial cells of the filter chamber encodes an aquaporin, consisting of 255 amino acids $(26 \mathrm{kDa})$, which has been named AQPcic. mRNA for this protein was injected into $X$. laevis oocytes to determine the kind of solute that can be transported through this water channel [18]. In comparison with the control, water transport through the oocyte membrane with injected mRNA for the aquaporin, increased 15-fold. This effect continued for 1-4 days after injection. AQPcic was not, however, permeable to glycerol. Expression in X. laevis indicates that mercury ions can inhibit water transport, but only at relatively high concentrations. This is likely an effect of the localization of cysteines in the aquaporin polypeptide chain. Normally, the cysteine in loop $\mathrm{E}$ is responsible for the inhibition of water permeability, however in AQPcic, this residue does not exist. Only Cys82 and Cys90 localized near the NPA motif in the loop B and Cys134 in the loop C could potentially be $\mathrm{Hg}^{+}$-binding sites. Because these cysteines are localized near the transmembrane domains, access to them by ions is difficult. Therefore, a higher concentration of ions is most likely necessary to inhibit water permeability [18] (Fig. 3). 


\section{Aquaporins localized in Drosophila melanogaster}

In the Drosophila melanogaster genome, six genes have been identified which encode proteins forming water channels. These proteins have been named: DRIP, Aqp4019, Aqp17664, Aqp7777, Aqp17662, and Aqp5398 [15]. The first four occur in both embryonic and adult stages. The localization of Aqp17662 and Aqp5398 is unclear.

In the embryonic stage of D. melanogaster, Aqp7777 is expressed in the brain and in the segmental ganglia. Aqp17664 is localized in the salivary glands and in a part of the gut. Aqp4019 exists in the body wall and visceral muscles. DRIP is expressed in many tissues throughout development, even can be localized in the early stages to the blastoderm. This means that the transcript of DRIP already occurs in the oocyte and thus, the appearing protein evidently comes from mother. In the later stages of the insect's development, the water channel protein is localized in the midgut and hindgut, and later appears in a pharynx and at the foregut-midgut boundary. Finally, DRIP appears in posterior spiracles. The presence of DRIP in the early embryonic stage indicates that this protein is important for early development as well as for adults. For example, in the different gut segments this protein plays different roles. In the foregut, water secretion helps lubricate a food passage. In the midgut, aquaporins aid in nutrient uptake and in the hindgut, they allow for water reabsorption [15]. The localization of aquaporins near the posterior spiracles, suggest that water channels regulate the opening of the spiracles. Hereby, aquaporins can limit water vapor from the tracheole system [15].

Furthermore, in the imago stage of D. melanogaster development, DRIP is localized in the Malpighian tubules as well as in the gut [15]. Dissolved in water, uric acid salts are transported from the tubules to the gut. In the hindgut, water is recovered and transported back to coelom and uric acid crystals are excreted. In both organs, water is moved through the water channels.

In addition to DRIP, only Aqp4019 and Aqp17664 have been identified in the imago stage. They occur with DRIP in the Malpighian tubules, however, unlike DRIP they are not in stellate cells but in the principal cells.

To date, only DRIP has been expressed in X. laevis oocytes [15]. The influence of this protein on the transport of water, glycerol, and urea has been examined. It has been observed that DRIP increases water permeation through the plasma membrane. One of the common features of the water channels is the exclusion of proton transport. The same situation has been shown for DRIP. This protein transports only water, suggesting that DRIP belongs to the group of primarily water selective aquaporins, not to the aquaglyceroporin group. The influence of mercury ions $\left(\mathrm{Hg}^{2+}\right)$ on water transport has not yet been investigated. The localization of cysteines within and adjacent to the transmembrane domains, implies that $\mathrm{Hg}^{2+}$ can not bind to these residues (Fig. 3).

Considering the amino acid sequence, it would be expected that the high transport selectivity is the result of two uncharged residues at positions 196 and 
200, localized just before the NPA motif. In addition, positions 212 and 213 are tyrosine and tryptophan which are typical aromatic acids in primarily water selective aquaporins in mammals. A lack of amino acids responsible for glycerol transport in loop $\mathrm{C}$ confirms that this aquaporin is not capable of permeating this solute. However, these sequences have been found in Aqp4019(GLCVT), Aqp17664(GVCLT), Aqp17662(GVCVT), and in Aqp5398(GICLV) which suggests that these proteins belong to the aquaglyceroporins group [15].

As with all proteins belonging to the aquaporin family, the aquaporins of D. melanogaster also have six transmembrane domains, however, the NPA motif is not conserved in all characterized proteins [15]. In Aqp5398, the NPC motif occurs in loop B (Asn-Pro-Cys), in Aqp17664 it is the NPV motif (Asn-Pro-Val), while in Aqp17662 the NPT motif (Asn-Pro-Thr) occurs.

\section{AQUAPORINS OF NEMATODE}

\section{Aquaporins localized in Caenorhabditis elegans}

Analysis of the genome sequence indicates that in C. elegans, eleven aquaporins can be found [12], eight of which have been characterized: F32A5.5 (AQP-1), C01G6.1 (AQP-2), Y69E1A.7 (AQP-3), F40F9.9 (AQP-4), C35A5.1 (AQP-5), C32C4.2 (AQP-6), M02F4.8 (AQP-7), and K02G10.7 (AQP-8). Among these proteins, $\mathrm{AQP}-4, \mathrm{AQP}-5$, and $\mathrm{AQP}-6$ are primarily water selective aquaporins, while AQP-1, AQP-2, AQP-3, AQP-7, and AQP-8 are aquaglyceroporins [12].

It has been established through the expression of aquaporins ( AQP-1 AQP-8) in X. laevis oocytes, that water channels built by AQP-2, AQP-3, AQP-4, AQP-6, and AQP-7 increase water transport through the cell membrane 5-7-fold when compared to the control oocytes. Furthermore, transport through AQP-4, AQP-6, and AQP-7 is inhibited by mercury ions. Analysis of AQP-4 revealed the reason behind this inhibition. Only one (Cys132), of the four cysteines which potentially could bind $\mathrm{Hg}^{2+}$, was a mercury-binding site. This cysteine residue was mutated to tryptophan and threonine, resulting in a lack of inhibition by mercury ions and a decrease in water transport. The replacement of the cysteine residue by threonine decreased the rate of transport to only 1.5 -fold faster than the control oocytes [16]. It was shown however, that AQP-2 and AQP-3 are mercury insensitive.

An analysis of amino acid sequences indicates that AQP-2 and AQP-4 have potential phosphorylation sites. For AQP-2 this is Ser69 for kinase A as well as Ser69 and Thr280 for kinase C. AQP-4 has four potential sites for kinase C; Thr28, Thr35, Ser36, Thr235, and one for kinase A, Ser33 [16, 17]. Analysis of AQP-8 shows that this protein has two variants. The smaller consists of 258 amino acids, while the larger has 294. It is likely that the first has five transmembrane 
domains, while the second has six. A similar situation characterizes human AQP10, which also has two isoforms. However, unlike AQP-8 with a shorter nonfunctional variant, that does not form a water channel, this protein is functional in both forms [20].

To define the role of aquaporins identified in C. elegans, the location of their expression has been studied [12]. One of the most typical places is within the excretory system, where in worms three aquaporins; AQP-2, AQP-3, and AQP-8 were identified. Cells containing these proteins are responsible for water excretion and secretion. AQP-8 can also be found in glands belonging to this system, AQP-2 is commonly found in muscles, motor neurons and in the hypodermis which plays an important role in maintaining a correct osmotic balance. AQP-3 has also been identified in male seminal vesicles and in the vas deferens.

AQP-1 and AQP-4 are responsible for water absorption in the intestine. AQP-4 is localized in an apical membrane, whereas AQP-1 is found in a basolateral membrane of the intestine epithelial cells [12]. It is connected with an adaption to hypertonic stress. Glycerol is important in the prevention of stress effects. A major site of glycerol production is the intestine. During hypertonic stress, AQP-1 may promote the efflux of glycerol to the pseudocoelomic fluid which can in turn, transfer this solute to all cells.

Expression of AQP-7 is periodic and is found only in muscles, while AQP5 and AQP-6 was observed in the neurons [12]. AQP-5 is expressed in neurons which are likely to correspond with pharyngeal interneurons. During the absence of food, these neurons modulate the rate of pharyngeal pumping. The other, AQP-6, is localized in sensory neurons responsible for food foraging behaviors [12].

Research has shown that the expression of aquaporins is not essential for organism functioning, nor for the maintainance of correct osmotic balance. Mutants lacking the AQP-2, AQP-3, AQP-4, and AQP-8 proteins exhibit normal development, life span, and fertility. Despite these water channels having high water permeability, a lack of them did not affect organism functionality and adaptation to hypertonic stress [12].

3.2 Aquaporin identified in Toxocara canis

Expression of the aquaporin gene $T c$-aqp-1, occurs in both larvae and adult males and females. mRNA-encoding aquaporin was found in the head, hypodermic/underlying muscles, and ovaries of the adult. However, the physiological function of this protein is still unclear. It is probable that it plays a part in forming a hydrostatic skeleton necessary for locomotion. Unfortunately, the expression in X. laevis oocytes was unsuccessful, and thus investigation of this protein was impossible. Nevertheless, established cDNA of Tc-aqp-1 was found to consist of $1099 \mathrm{bp}$, however $21 \mathrm{bp}$ from 5'and $147 \mathrm{bp}$ from 3' remain as untranslated regions. The predicted protein was estimated to contain 310 amino acids $(34.3 \mathrm{kDa})$. It forms six transmembrane domains and possesses one potential $\mathrm{N}$-glycosylation site (Asn153) [19]. 


\section{COMPARISON INVERTEBRATE AQUAPORINS WITH MAMMALIAN AQUAPORINS}

There are noticeable similarities in structure and function between characterized invertebrate aquaporins and their counterparts in mammals. Bioinformatic research has reported that identified to date invertebrate water channels show the highest correlation with some mammalian aquaporins, namely AQP4 and AQP1 (Table 1).

\section{Comparison of invertebrate aquaporins with the mammalian aquaporin AQP4}

Some invertebrate aquaporins reveal the highest amino acid identity with mammalian AQP4. This group includes; AeaAQP from A. aegypti with a sequence $48 \%$ identical to mouse AQP4, and DRIP from D. melanogaster which has a sequence 44\% identical to human AQP4 [16]. Correlation between AeaAQP and AQP4 goes beyond the amino acid sequence. The localization of both proteins are associated with the respiratory system, although the mRNA for these proteins are not restricted to this region [24, 27]. AeaAQP can be transcribed in other cells, where water channels do not exist. A similar situation is found in the rodent, Dipodomys merriami merriami, where AQP4 is localized in respiratory system but the mRNA can also occur in the kidney [13].

Another common feature between invertebrate aquaporins and mammalian AQP4 is in the formation of plasma membranes crystalline orthogonal arrays of particles (OAPs) [26]. Both AeaAQP and AQP4 occur in the plasma membranes, only within these structures. Particle spacing within OAPs in invertebrates and mammals is very similar, measuring $6.7 \mathrm{~nm}$ long for AeaAQP and $6.8 \mathrm{~nm}$ for AQP4 [7, 29].

An important distinction between the water channels built by AeaAQP and by AQP4 is the influence of the mercury ions. Flow through a water pore built by Aea AQP can be blocked by $\mathrm{Hg}^{2+}$ while a water pore built by AQP4 is insensitive to these ions [9]. Distinguishing factors between these aquaporins is a lack of asparagine in position 153 in AeaAQP which is a N-glycosylation site in AQP4. In AQP4 there is a phosphorylation site in loop B, which does not exist in AeaAQP [20].

The most similarities in structure and function exist between DRIP and AQP4. Both are primarily water selective and do not conduct another solutes. Transport is very fast and in $X$. laevis oocytes, water flux is increased approximately 10 -fold. It is likely that DRIP, like AQP4, is $\mathrm{Hg}^{2+}$ insensitive [15]. 


\begin{tabular}{|c|c|c|c|c|c|}
\hline 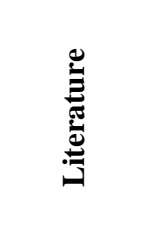 & 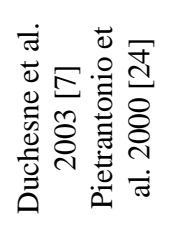 & 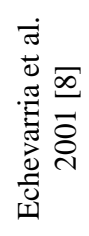 & 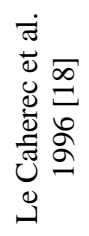 & 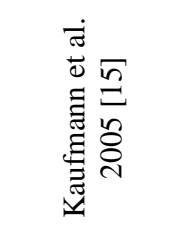 & 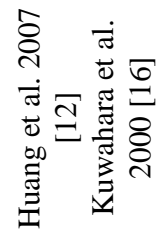 \\
\hline 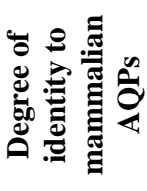 & 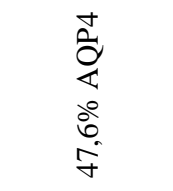 & $\begin{array}{l}\bar{a} \\
\stackrel{\Delta}{0} \\
\dot{o} \\
i\end{array}$ & 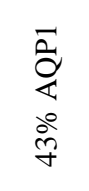 & 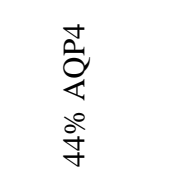 & 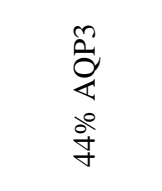 \\
\hline 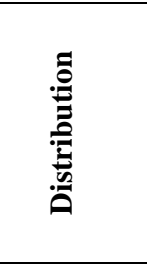 & 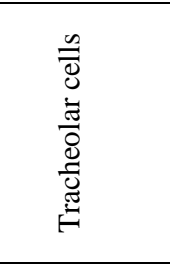 & 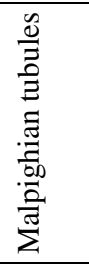 & 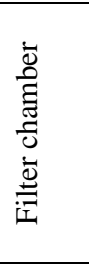 & 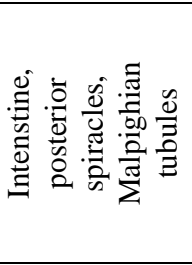 & 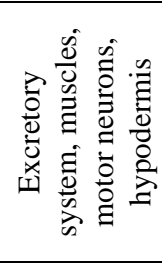 \\
\hline 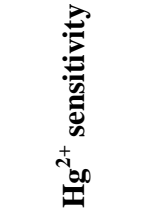 & $\stackrel{0}{\nu}$ & $\stackrel{\circ}{z}$ & $\overbrace{\nu}^{\infty}$ & 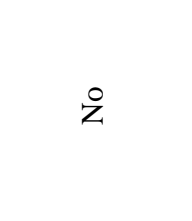 & $\stackrel{\circ}{z}$ \\
\hline 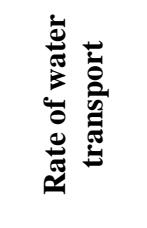 & $\begin{array}{l}\frac{0}{0} \\
\stackrel{0}{0} \\
0\end{array}$ & $\begin{array}{l}\frac{\pi}{0} \\
\stackrel{d}{d} \\
\text { d }\end{array}$ & $\begin{array}{l}\frac{7}{0} \\
\stackrel{1}{n} \\
\text { (n) }\end{array}$ & $\begin{array}{l}\frac{\pi}{0} \\
\stackrel{1}{0} \\
0\end{array}$ & $\frac{0}{0}$ \\
\hline : & $\begin{array}{l}\frac{\mathbb{v}}{\pi} \\
\frac{\pi}{3}\end{array}$ & $\begin{array}{l}\frac{\vec{D}}{\tilde{N}} \\
\frac{1}{3}\end{array}$ & $\begin{array}{l}\overline{\mathbb{D}} \\
\frac{\pi}{3}\end{array}$ & 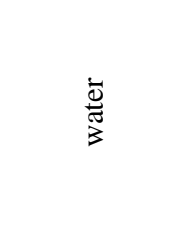 & 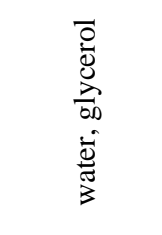 \\
\hline 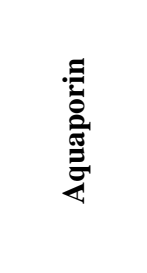 & 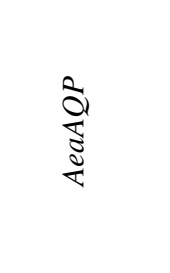 & 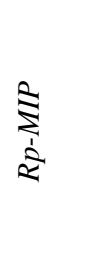 & 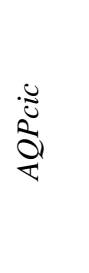 & $\vec{\Xi}$ & 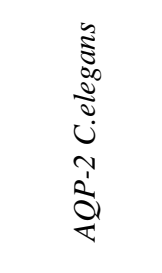 \\
\hline
\end{tabular}




\section{Comparison of invertebrate aquaporins with mammalian aquaporin AQP1}

Within invertebrate aquaporins, only Rp-MIP and AQPcic reveal similarity to AQP1 $[8,18]$.

An Rp-MIP amino acid sequence is $26 \%$ identical to mammalian AQP1. The localization of these proteins is also similar. Both occur in organs responsible for forming and excreting urine. However Rp-MIP differs from AQP1 in some ways. AQP1 can transport water at a considerable rate but this transport is inhibited by mercury ions [32]. Rp-MIP increases water permeability only 2 -fold, and this transport can not be inhibited by mercury ions [8].

As compared to Rp-MIP, AQPcic from $C$. viridis has more in common with AQP1. Firstly, its amino acid sequence is $43 \%$ identical to AQP1 [7]. The rate of the water transport through AQPcic is faster than through Rp-MIP, but is not as fast as through AQP1 [18]. Water permeability can be inhibited by mercury ions, but the cause of this phenomenon is unclear. In AQP1, the Cys189 in loop E is an ion-binding residue, while in AQPcic, Cys82 and Cys90 in loop B and Cys135 in loop $\mathrm{C}$ perform this function. This has an influence on the $\mathrm{Hg}^{2+}$ concentration needed to inhibit water transport. In the case of AQPcic, 3-4 fold more $\mathrm{Hg}^{2+}$, is required for this inhibition as compared with AQP1 [18].

\section{SUMMARY}

All invertebrate aquaporins share typical features with other proteins belonging to the aquaporin family. They transport water, as well as in some cases, glycerol. Their polypeptide chain sequences contain six transmembrane domains and generally two NPA motifs within the water pore. Furthermore, both polypeptide ends are localized at the cytoplasmic site.

A comparison of the structure, location and function of the identified invertebrate aquaporins, with their counterparts in well characterized vertebrate groups of aquaporins, show common features of aquaporins across both the groups of animals.

Characterization of mammalian aquaporins increases our knowledge about the variety of functions of water channels. Exploring invertebrate aquaporins could extend this knowledge.

It is clear that this is just the beginning of studies into invertebrate aquaporins. As compared with water channels of vertebrates, only few invertebrate aquaporins have been characterized to date. Considering the enormous variety of species and diverse adaptations to the environment, within the insects group alone, it could be expected that the number of aquaporins could be large and their functions may be difficult to predict. Identification and characterization of these proteins could be very useful in practice [33]. For example, identifying aquaporins 
of the protozoan parasites, Plasmodium falciparum has allowed researchers to determine that inhibitors of these proteins may be used to eliminate these parasites $[2,3,4]$.

\section{REFERENCES}

[1] AGRE P. Aquaporins water channels. Angew Chem Int Ed 2004 43: 4278-4290

[2] BAHOMONTES- ROSA N, BINGUHA W, BEITZ E, KREMSNER PG, KUN JFJ. Limited genetic diversity of the Plasmodium falciparum aquaglyceroporin gene. Mol Biochem Parasitol 2007 156: 255-257

[3] BAHOMONTES- ROSA N, ROBIN A, AMBROSIO AR, MESSIAS-REASON I, BEITZ E, FLITSCH SL, KUN JFJ. Monoguaternary ammonium derivatives inhibit growth of protozoan parasites. Parasitol Int 2008 57: 132-137

[4] BEITZ E. Aquaporins from pathogenic protozoan parasites: structure, function and potential for chemotherapy. Biol Cell 2005 97: 373-383

[5] CAMPBELL EM, BALL A, HOPPLER S, BOWMAN AS. Invertebrate aquaporins: a review. $J$ Comp Physiol B 2008 178: 935-955

[6] CASTLE NA. Aquaporins as targets for drug discovery. Drug Discov Today 2005 10: 485-493

[7] DUCHESNE L, HUBERT JF, VERBAVATZ JM, THOMAS D, PIETRANTONIO PV. Mosquito (Aedes aegypti) aquaporin present in tracheolar cells, transports water not glycerol and forms orthogonal arrays in Xenopus oocyte membranes. Eur J Biochem 2003 270: 422-429

[8] ECHEVARRIA M, REMIREZ-LORCA R, HERNANDEZ CS, GUTIERREZ A, MENDEZ FERRER S, GONZALEZ E, TOLEDO-ARAL JJ, ILUNDAIN AA, WHITTEMBURY G. Identification of a new water channel (Rp-MIP) in the Malpighian tubules of the insect Rhodnius prolixus. Pflügers Arch - Eur J Physiol 2001 442: 27-34

[9] FU L, LU M. The structural basis of water permeation and proton exclusion in aguaporins (Review). Mol Membr Biol 2007 24: 366-374

[10] GONEN T, WALZ T. The structure of aquaporins. Q Rev Biophys 39: 361-396

[11] HOHMANN J, BILL RM, KAYINGO J, PRIOR BA. (2000) Microbial MIP channels. Trends Microbiol 2006 8: 33-38

[12] HUANG CG, LAMITINA T, AGRE P, STRANGE K. Functional analysis of the aguaporin gene family in Caenorhabditis elegans. Am J Physiol Cell Physiol 2007 292: C1867-C1873

[13] HUANG Y, TRACY R, WALSBERG GE, MAKKINJE A, FANG P, BROWN D, VAN HOCK AN. Absence of aquaporin-4 water channels from kidneys of the desert rodent Dipodomys merriami merriami. Am J Physiol Renal Physiol 2001 280: F794-802

[14] KALDENHOFF R, FISCHER M. Aquaporins in plants. Acta Physiol 2006 187:169-176

[15] KAUFMANN N, MATHAI JC, HILL WG, DOW JAT, ZEIDEL ML BRODSKY JL. Developmental expression and biophysical characterization of a Drosophila melanogaster aquaporin. Am J Physiol Cell Physiol 2005 289: C397-C407

[16] KUWAHARA M, ASAI T, SATO K, SHINBO I, TERADA Y, MARUMO F, SASAKI S. Functional characterization of a water channel of the nematode Caenorhabditis elegans. Biochim Biophys Acta 2000 1517: 107-112

[17] KUWAHARA M, ISHIBASHI K, GU Y, TERADA Y, KOHARA Y, MARUMO F, SASAKI S. A water channel of the nematode $C$. elegans and its implications of channel selectivity of MIP proteins. Amer J Phys Cell Phys 1998 275: C1459-C1464

[18] LE CAHEREC F, DASCHAMPS S, DELAMARCHE C, PELLERIN I, BONNEC G, GUILLAM MT, THOMAS D, GOURANTON J, HUBERT JF. Molecular cloning and characterization of an insect aquaporin. Functional comparison with aquaporin 1. Eur J Biochem 1996 241: 707-715

[19] LOUKAS A, HUNT P, MAIZELS RM. Cloning and expression of an aquaporin-like gene from a parasitic nematode. Mol Biochem Parasitol 1999 99: 287-293 
[20] MAH AK, ARMSTRONG KR, CHEW DS, CHU JS, TU DK, JOHNSEN RC, CHEN N, CHAMBERLIN HM, BAILLIE DL. Transcriptional regulation of AQP-8, a Caenorhabditis elegans aquaporin exclusively expressed in the excretory system, by the POU homeobox transcription factor CEH-6. J Biol Chem 2007 282: 28074-28086

[21] MARTINEZ AS, CUTLER CP, WILSON GD, PHILLIPS C, HAZON N, CRAMB. Cloning and expression of three aquaporin homologues from the European eel (Anguilla anguilla): effects of seawater acclimation and cortisol treatment on renal expression. Biol Cell 2005 97: 615-627

[22] MORISHITA Y, SAKUBE Y, SASAKI S, ISHIBASHI K. Molecular mechanisms and drug development in aquaporin water channel diseases: Aquaporin superfamily (superaquaporins): Expansion of aquaporins restricted to multicellular organisms. J Pharmacol Sci 2004 96: 276-279

[23] MURATA K, MITSUOKA K, HIRAL T, WALZ T, AGRE P, HEYMANN JB, ENGEL A, FUJIYOSHI Y. Structural determinants of water permeation through aquaporin-1. Nature 2000 407: 599-605

[24] PIETRANTONIO PV, JAGGE C, KEELEY LL, ROSS LS. Cloning of an aquaporin-like cDNA and in situ hybridization in adults of the mosquito Aedes aegypti (Diptera: Culicidae). Insect Mol Biol 2000 9: 407-418

[25] RAMIREZ-LORCA R, MUNOZ-CABELLO AM, TOLEDO-ARAL JJ, ILONDAIN AA, ECHEVARRIA M. Aquaporins in chicken: localization of ck-AQP5 along the small and large intestine. Comp Biochem Physiol A Mol Integr Physiol 2006 143: 269-277

[26] RASH JE, YASUMURAT, HUDSON CS, AGRE P, NIELSEN S. Direct immunogold labeling aquaporin-4 in square arrays of astrocyte and ependymocyte plasma membranes in rat brain and spinal cord. Proc Natl Acad Sci U S A 1998 95: 11981-11986

[27] SONG Y, JAYARAMAN S, YANG B, MATTHAY MA, VERKMAN AS. Role of aquaporin water channel in airway fluid transport. J Gen Physiol 2001 117:573-582

[28] SUZUKI M, HASEGAWA T, OGUSHI Y, TANAKA S. Amphibian aquaporins and adaptation to terrestrial environments: a review. Comp Biochem Physiol A Mol Integr Physiol 2007 148: 72-81

[29] TAKATA K, MATSUZAKI T, TAJIKA Y. Aquaporins: water channel proteins of the cell membrane. Prog Histochem Cytochem 2004 39: 1-83

[30] TANGHE A, VAN DIJCK P, THEVELEIN JM. Why do microorganisms have aquaporins? Trends Microbiol 2006 14: 78-85

[31] WANG Y, TAJKHORSHID. Molecular mechanisms of conduction and selectivity in aquaporin water channels. J Nutr 2007 137: 1509S-1515S

[32] WU B, BEITZ E. Aquaporins with selectivity for unconventional permeants. Cell Mol Life Sci 2007 64: 2413-2421

[33] YOOL AJ. Functional domains of aquaporin-1: Keys to physiology, and targets for drug discovery. Curr Pharm Des 2007 13: 3212-3221

[34] ZELENINA M, ZELENIN S, APERIA A. Water channels (aquaporins) and their role for postnatal adaptation. Pediatr Res 2005 57: 47R-53R

Corresponding author: Joanna Romana Pienkowska

Department of Cell Biology, Faculty of Biology, Adam Mickiewicz University

89 Umultowska Street, 61-614 Poznan, Poland

e-mail:pienkowj@amu.edu.pl 